\title{
A Quick Fault Detection System Applied to Pitch Actuators of Wind Turbines
}

\author{
L. Acho $^{1}$ \\ ${ }^{1}$ Department of Mathematics, \\ Polytechnic University of Catalunya. \\ Campus Terrassa - C. Colom-2, 08222 Terrassa (Spain) \\ Phone/Fax number:+0034 630581156, e-mail: leonardo.acho@upc.edu
}

\begin{abstract}
The design of fast respond fault detection systems to wind turbines results an important subject and represents a notable challenger too. This paper presents a recent approach on a quick response fault detection system to pitch actuators in controlled wind turbines. The obtained time detection is about 10 seconds. Our scheme was possible by manipulating an adaptive parametric estimation block by varying the time scales among the actuator and the identification process dynamics. Additionally, numerical experiments are realized to support the main contribution.
\end{abstract}

Key words. Wind turbines, on-line fault detection, adaptive dynamics.

\section{Introduction}

Nowadays, it is well recognized the benefit impacts of renewable energies in ours and future life on earth and on other planets too, as Mars will be in the near future [1][2]. To assure an efficient operation on energy conversion of these renewable systems, an efficient and editable response fault detection system is a key factor [3]-[4]. Among the different renewable energy approaches, the wind turbines operate with electro-mechanical parts in comparison to, for instance, photovoltaic developments. Therefore, a mechanical fault detection algorithm is mandatory. Hence, the main objective of this paper is to propose a programmed adaptation gain in an adaptive identification scheme to develop an efficient fault detection method to pitch actuators in controlled wind turbines. In literature, there are many contributions on it. Some are based on data analysis [5], others are based on dynamical observers [6], and so on [7]. On the other hand, adaptive identification techniques can be tracked from the 70s [8], since then, it is a well-studied topic [9]. In the adaptive identification domain, the most used methods are [8]: 1) The gradient algorithm, 2) the recursive leastsquares technique, 3) the model reference identification approach, and 4) the normalized gradient process with projection. For instance, the recursive least-squares approach is a good technique, but its algorithm does not comprise an adaptation gain on its adaptation mechanism [8]. Here, by using a time-scale transformation, this gain parameter can be trivially added to this identification process and then accelerating the parametric identification time. Actually, this innovation allows us to develop our identification process technique. When this development is applied to the fault detection objective in pitch actuators of wind turbines, its performance is notorious.

The rest of the paper is structured as follows. Section 2 gives a brief on adaptive parametric on-line identification, the recursive least-squares algorithm, along with our contribution by adding it the adaptation gain to tune the convergence speed of the identification process. Section 3 describes the problem statement on fault detection in pitch actuator systems of wind turbines. Section 4 describes our main contribution followed by numerical experiments. According to these experiments, a fault can be detected between 1 to 10 seconds. Finally, Section 5 presents our main conclusions.

\section{Adaptive parametric on-line identification}

The recursive least-squares algorithm for parametric identification uses the following performance index [8]:

$$
\mathrm{J}=\int\left[\theta^{T}(t) w(\tau)-y_{p}(\tau)\right]^{2} d \tau
$$

where $\theta(t) \in \mathbb{R}^{p}$ is the on-time estimation vector of the parametric plant, $w(t) \in \mathbb{R}^{q}$ is the regression vector, and $y_{p} \in \mathbb{R}$ is the plant output given by :

$$
y_{p}(t)=\theta^{* T} w(t)
$$

being $\theta^{*} \in \mathbb{R}^{p}$ the nominal system vector parameter. Then, the recursive least-squares algorithm is stated as follows [8]:

Theorem 1.- The following adaption dynamics minimize the performance index in equation (1):

$$
\begin{gathered}
\dot{P}(t)=-P(t) w(t) w^{T}(t) P(t), \\
\theta(t)=-P(t) w(t)\left[w^{T}(t) \theta(t)-y_{p}(t)\right] .
\end{gathered}
$$

Moreover, if the excitation vector signal $w(t)$ is a persistent excitation class, then $\theta(t)$ converges to $\theta^{*}$ as time goes on. 
Remark 1.- The standard notation has been used. That is, the dot notation (') represents derivation with respect to time. Additionally, observe that $P(t) \in \mathbb{R}^{q \times q}$.

The main drawback of the above principal result is the missing adaptation gain parameter. This parameter is common in adaptive parametric algorithms to tune the estimation speed of the parametric identification process. This is so important to adequate the system identification dynamic to the plant time response [10]. A way to include this gain parameter to the recursive least-squares algorithm is by realizing a time-scale transformation: $=g t$. Therefore, we arrive to the next evident result:

Proposition 1.- The following alternative on adaption dynamics minimize the performance index in equation (1):

$$
\begin{gathered}
\dot{P}(t)=-g_{1} P(t) w(t) w^{T}(t) P(t), \\
\theta \dot{(t)}=-g_{2} P(t) w(t)\left[w^{T}(t) \theta(t)-y_{p}(t)\right] .
\end{gathered}
$$

Additionally, if the excitation vector signal $w(t)$ is a persistent excitation class, then $\theta(t)$ converges to $\theta^{*}$ as time goes on. Now, the real parameters $g_{1}$ and $g_{2}$ independently control the transient responses of the systems in (5)-(6), respectively.

Remark 2.- The dot notation (') also stand for derivation with respect to time even when we realized a time-scale transformation, for simplicity notation.

Remark 3.- The parameters $g_{1}$ and $g_{2}$ are introduced to manipulate different time scales in the dynamics stated in (5)-(6). These time scales manipulation strategy gives us the option to correctly drive the systems to easily detect faults on a given system, as it is evidenced later. This is the main key of our approach.

\section{Problem statement}

Usually, the blade pitch system of wind turbines consists of three identical independent pitch actuators governed by using a PI controller each one [3], [4], [11]. Moreover, the dynamical model of a pitch actuator wind turbine can be captured by using the next mathematical model [3],[4],[11]:

$$
\frac{\beta(s)}{\beta_{r e f}(s)}=\frac{\omega_{n}^{2}}{s^{2}+2 \zeta w_{n} s+\omega_{n}^{2}},
$$

where $\beta(t)=y_{p}(t)$ is the pitch angle related to its actuator and represents the plant output, $\beta_{\text {ref }}(t)$ is the reference command supplied by the control power management system. Additionally, $w_{n}$ is the natural frequency, and $\zeta$ is the damping ratio of the pitch actuator mechanism. In hydraulic pitch actuators, its degradation performance comes from different scenarios such as pump wear, hydraulic leakage, and high air oil content [3-5]. Obviously, these scenarios may affect the actuator behaviour leading to dangerous faults. From the plant parametric point of view, these faulty stages can be parametrically captured. See Table I. Therefore, the problem statement consists to design a quick fault detection system for a pitch actuator mechanism in wind turbines by using the input-output information of the plant. It is so important to note that the maximum blade pitch rate in wind turbines is usually stated as $\pm 8 \mathrm{deg} / \mathrm{s}$ [3]. Later on, in our experimental results, this fact is satisfied.

Table 1.- Parameters for hydraulic pitch system under common faulty scenarios $[4,11]$.

\begin{tabular}{|c|c|c|}
\hline Scenario & $\begin{array}{c}\text { Parameter } \\
\omega_{n} \\
(\mathrm{rad} / \mathrm{s})\end{array}$ & $\begin{array}{c}\text { Parameter } \\
\zeta\end{array}$ \\
\hline No fault $(H)$ & 11.11 & 0.6 \\
\hline High air oil content $\left(F_{1}\right)$ & 5.73 & 0.45 \\
\hline Hydraulic leakage $\left(F_{2}\right)$ & 3.42 & 0.9 \\
\hline Pump wear $\left(F_{3}\right)$ & 7.27 & 0.75 \\
\hline
\end{tabular}

\section{Fault detection design and numerical experiments}

This section presents our fault detection approach by using the result presented in Proposition 1. Additionally, and in order to obtain a reduced fault detection scheme, we are going to use, just for design purposes, the following reduced model of the pitch actuator model [3]:

$$
\dot{\beta}(t)=-a_{1} \beta(t)+a_{2} \beta_{r e f}(t),
$$

where $a_{1}$ and $a_{2}$ are the model actuator parameters.

The overall fault detection system is based on the information signals coming from the input and output of the pitch actuator system. See Figure 1. In this Figure, the proposed on-line parametric estimation process employs the filtering signals of the input/output of the actuator device (then, the parameter $\lambda$ is known).

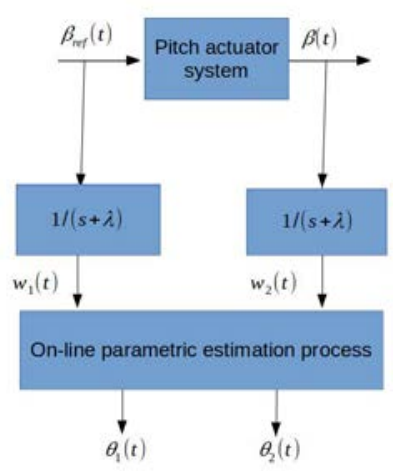

Fig. 1.- General representation of the fault detection system. 
From the system in equation (8), its frequency-domain by using Laplace transformation gives:

$$
s \beta(s)=-a_{1} \beta(s)+a_{2} \beta_{r e f}(s),
$$

which we may manipulate to produce:

$$
\frac{s \beta(s)}{s+\lambda}=\frac{-a_{1} \beta(s)}{s+\lambda}+\frac{a_{2} \beta_{r e f}(s)}{s+\lambda} .
$$

The above is then equivalent to:

$$
\beta(s)=\frac{\left(\lambda-a_{1}\right) \beta(s)}{s+\lambda}+\frac{a_{2} \beta_{r e f}(s)}{s+\lambda} .
$$

Then, and in correlation to Figure 1, we have:

$$
\beta(s)=\left(\lambda-a_{1}\right) w_{2}(s)+a_{2} w_{1}(s) .
$$

In matrix notation, the above yields:

$$
\underbrace{\beta(s)}_{y_{p}(s)}=\underbrace{\left[\begin{array}{ll}
a_{2} & \left(\lambda-a_{1}\right)
\end{array}\right]}_{\theta^{T}(s)} \underbrace{\left[\begin{array}{l}
w_{1}(s) \\
w_{2}(s)
\end{array}\right]}_{w(s)},
$$

where the system (13) converges to system (2) if $\theta(t)=$ $\left[\begin{array}{ll}\theta_{1}(t) & \theta_{2}(t)\end{array}\right]^{T}$ converges to $\theta^{*}=\left[\begin{array}{ll}a_{2} & \left(\lambda-a_{1}\right)\end{array}\right]^{T}$ as time goes on. That is, if $\theta_{1}(t) \rightarrow a_{2}$ and $\theta_{2}(t) \rightarrow\left(\lambda-a_{1}\right)$ as time goes on. Therefore, $\hat{y}_{p}(t) \rightarrow y_{p}(t)$. Hence, we have the following parametric estimation rules:

$$
\hat{a}_{2}(t)=\theta_{1}(t)
$$

and

$$
\hat{a_{1}}(t)=\lambda-\theta_{2}(t)
$$

Finally, and by following Proposition 1, Figures 2 to 9 present our numerical experiment results. In these experiments, we implement the dynamic actuator stated in (7) and by using the data displayed in Table 1 . Figures 2 and 3 show the dynamic results from the healthy stage to the faulty scenarios $F_{1}$ (red line), $F_{2}$ (blue line), and $F_{3}$ (green line) activated at $t=250$ seconds. But the yellow line corresponds to the healthy case all along the simulation time for comparison. This colour code labels will be kept in further results analysis. From these figures, it is clear that the faulty scenarios can be distinguished from the healthy stage. Hence, by using a reference healthy model, as in [4], a fault detection system would be straightforwardly realized. Figure 4 shows the used input signal to the system. On the other hand, Figures 5 and 6 show the system output for all cases, where from these signals, it is almost impossible to clarify the healthy case from the faulty ones. Furthermore, Figures 7 and 8 show another set of experiment results now by using the input signal shown in Fig. 9. The same conclusion can be reached. Additionally, from the previous results, we can note that a fault in Table 1 may be detectable in less than 10 seconds. Additionally, just one parameter observation is enough to conclude (we use $a_{1}(t)$ ). In these experiments, we use $g_{1}=10, g_{2}=1000, \lambda=5.87$,
$P(0)=I_{2 \times 2}$, and $\theta(0)=\left[\begin{array}{ll}0 & 0\end{array}\right]$. The value of $\lambda=$ 5.87 was obtained by using the healthy system parameters related to $\lambda=w_{n} \sqrt{1-2 \zeta^{2}}$ (the cut frequency of a second order system). Finally, to say that if $g_{1}=g_{2}=1$ (the standard adaptive parametric estimation algorithm), the fault detection system performance is totally degraded.

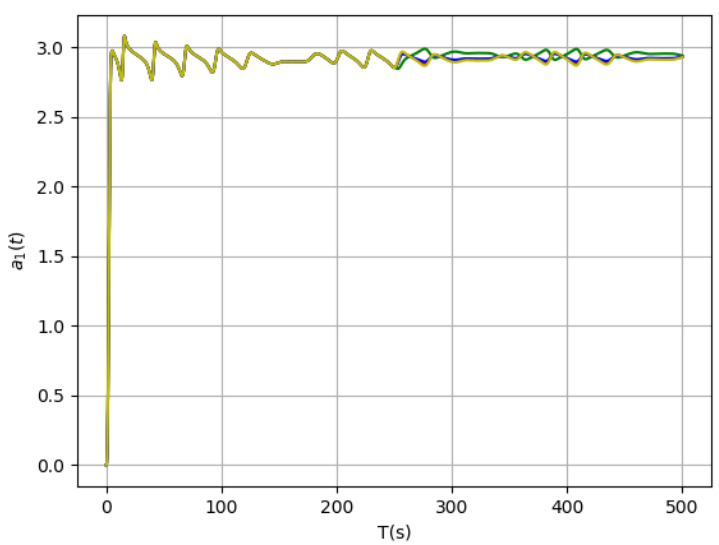

Fig. 2.- Simulation results for $a_{1}(t)$ from the healthy case to the fault scenarios activated at $t=250$ seconds but the yellow line which corresponds to the healthy case on along the simulation time for comparison.

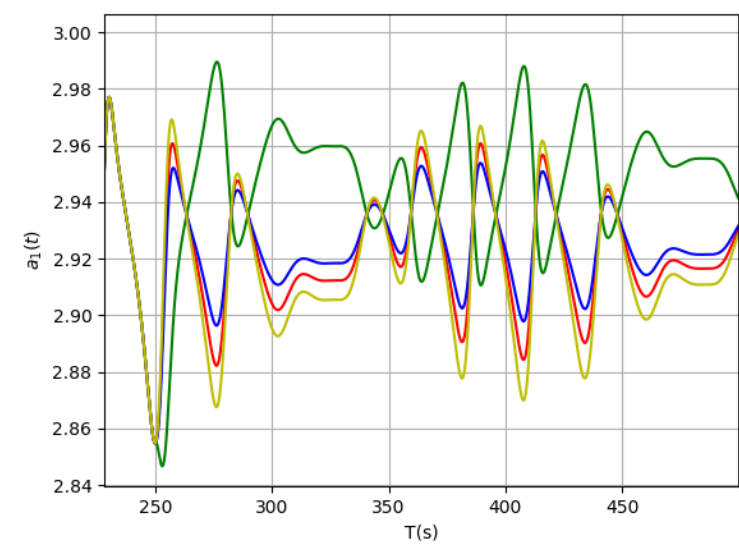

Fig. 3.- Simulation results: A zoom version of the picture on Fig. 2. 


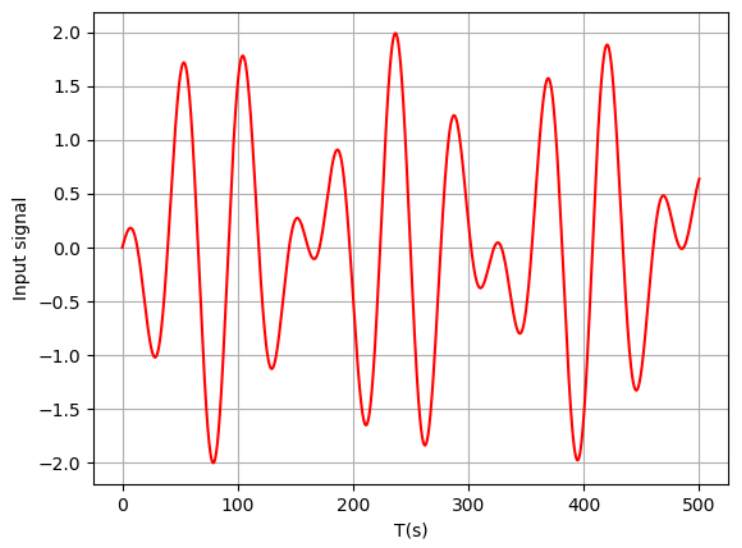

Fig. 4.- The employed input signal to the system.

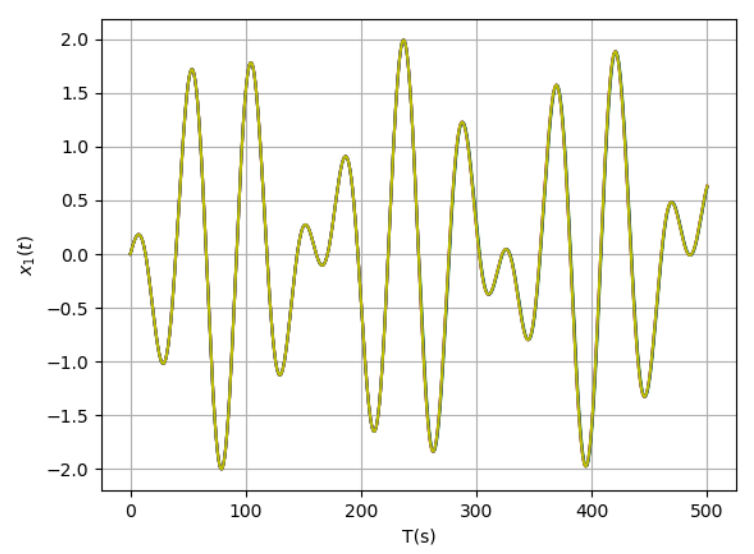

Fig. 5.- System output for all scenarios, the healthy and faulty cases $y_{p}(t)=x_{1}(t)$.

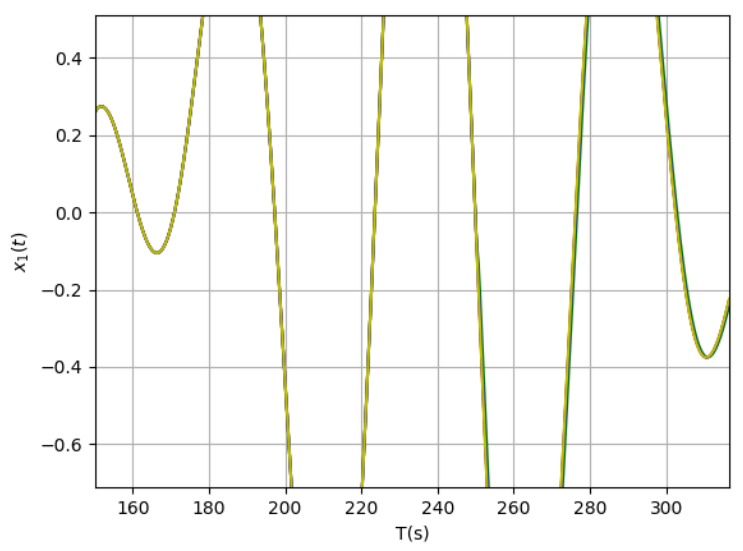

Fig. 6.- A zoom version of the picture on Fig. 5.

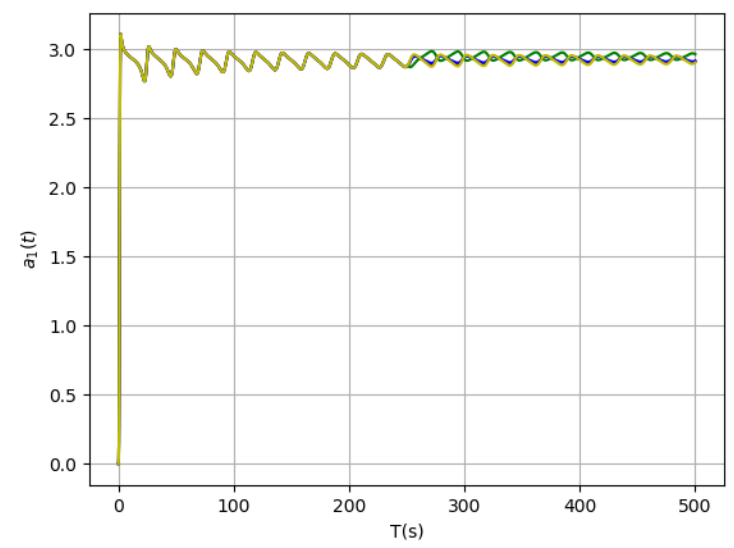

Fig. 7 .- Simulation results for $a_{1}(t)$ from the healthy case to the fault scenarios activated at $t=250$ seconds but the yellow line which corresponds to the healthy case on along the simulation time for comparison. Second example.

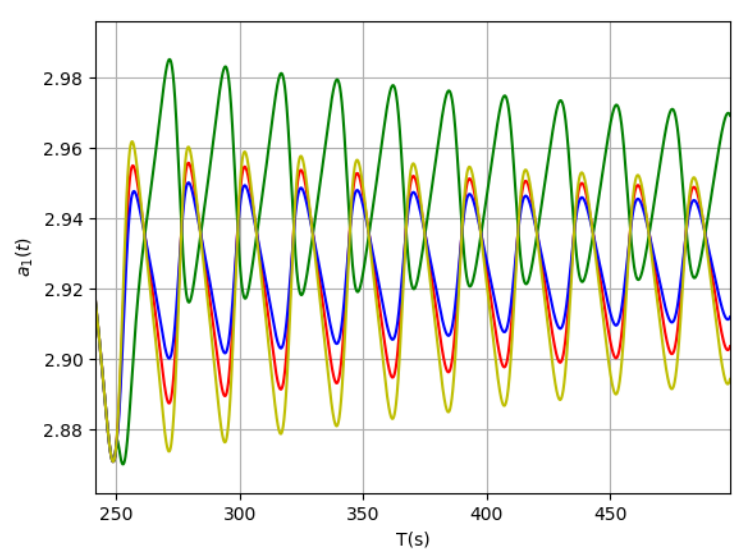

Fig. 8 .- Simulation results: A zoom version of the picture on Fig. 7. Second example.

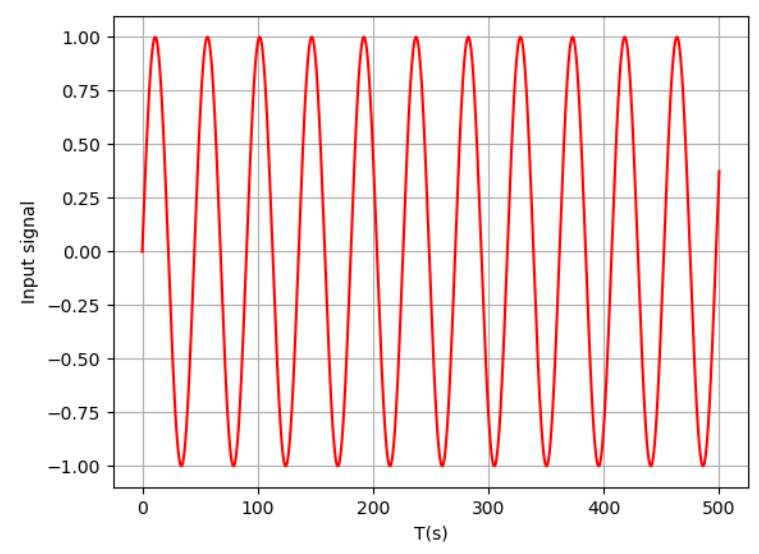

Fig. 9.- The employed input signal to the system. Second example. 


\section{Conclusion}

This paper has presented a recent approach on fault detection system to pitch actuators of wind turbines. This approach is based on manipulating the time scales among the actuator system of the wind turbine and the dynamic stages of the adaptive parametric estimation algorithm. Its main advantage is its quick response time.

\section{Acknowledgement}

This research was completely funded by the Spanish Ministry of Economy and Competitiveness/Fondos Europeos de Desarrollo Regional (MINECO/FEDER) grant number DPI2015-64170-R.

\section{References}

[1] D. Gielen, F. Boshell, D. Saygin, M. D. Bazilian, N. Wagner, R. Gorini, "The role of renewable energy in the global energy transformation”, Energy Strategy Reviews (2019). Vol. 24, pp. 38-50.

[2] A. Delgado-Bonal, F. J. Martín-Torres, S. Vazquez-Martín, M. P. Zorzano, "Solar and wind exergy potentials for Mars", Energy (2016). Vol. 102, pp. 550-558.

[3] N. Luo, Y. Vidal, L. Acho (Eds.), Wind Turbine Control and Monitoring, Springer Verlag, Germany (2014), pp. 301-334.

[4] M. Nazir, A. Q. Khan, G. Mustafa, M. Abid, "Robust fault detection for wind turbines using reference model-based approach”, Journal of King Saud University-Engineering Sciences (2017). Vol. 29(3), pp. 244-252.
[5] M. Ruiz, L. E. Mujica, S. Alferez, L. Acho, C. Tutiven, Y. Vidal, F. Pozo, "Wind turbine fault detection and classification by means of image texture analysis", Mechanical Systems and Signal Processing (2018). Vol. 107, pp. 149-167.

[6] P. F. Odgaard, J. Stoustrup, R. Nielsen, C. Damgaard, "Observer based detection of sensor faults in wind turbines", Proceedings of European Wind Energy Conference (2009), pp. 4421-4430.

[7] W. Qiao, D. Lu, "A survey on wind turbine condition monitoring and fault diagnosis-Part I: Components and subsystems", IEEE Transactions on Industrial Electronics (2015). Vol. 62(10), pp. 6536-6545.

[8] S. Sastry, M. Bodson, Adaptive control: stability, convergence and robustness, Courier Corporation, Massachusetts (2011), pp. 45-98 .

[9] N. Kalouptsidis, S. Theodoridis, S. (Eds.), Adaptive system identification and signal processing algorithms, Prentice Hall, New York (1993), pp. 7-81.

[10] H. M. Usman, S. Mukhopadhyay, H. Rehman, "Permanent magnet DC motor parameters estimation via universal adaptive stabilization”, Control Engineering Practice (2019). Vol. 90, pp. 50-62.

[11] S. Cho, Z. Gao, T. Moan, "Model-based fault detection, fault isolation and fault-tolerant control of a blade pitch system in floating wind turbines”, Renewable Energy (2018). Vol. 120, pp. 306-321. 